\title{
Gamma-Ray Polarimetry - Past Present and Future
}

\author{
A.J.Dean ${ }^{1}$ \\ School of Physics and Astronomy \\ The University of Southampton \\ Highfield \\ Southampton SO17 1BJ \\ E-mail: ajdeastro.soton.ac.uk
}

Astronomical polarimetric observations in the gamma-ray region of the spectrum are extremely difficult to achieve from a technical point of view. However there are great rewards to be gained since gamma-rays are mostly produced by non thermal processes related to highly ordered fields and particle beams close to the central engines that drive powerful cosmic sources. The intimate connection between the emitted gamma-ray fluxes and the central power sources ensures the measurement of their polarisation characteristics offers a powerful diagnostic for the discrimination between different source mechanisms whilst providing a unique insight into the geometrical and physical nature of the emission zone. The recent gamma-ray polarisation measurements of the Crab emission provide a powerful investigative tool for the physical conditions and geometry of the magnetic field close to the pulsar. The Crab nebula, has been found to emit linearly polarised gamma-rays during the off pulse phase with an efficiency close to the maximum allowable by physics. The close alignment between the electric vector and the spin axis of the neutron star places severe boundaries on theoretical models. The off pulse gamma-radiation is constrained to originate somewhere close to the pulsar, but outside the light cylinder. Three contenders are identified: the striped wind model; radiation from within the inner jet; Doppler boosted radiation from knot-like features close to the pulsar. In order to advance the field beyond a crop of measurements based on the strongest sources future polarimeters will require careful design in order to suppress systematic errors to an acceptable level, and will need a concentrator such as a Laué lens system in order to enhance the signal to noise level.

8th INTEGRAL Workshop "The Restless Gamma-ray Universe"

Dublin, Ireland

September 27-30, 2010

$1 \quad$ Speaker 


\section{Introduction}

The major sources of information relating to $\gamma$-ray sources has been derived from either spectroscopy or timing and provide fundamental details of source model scenarios, however ambiguities often remain, and the precise physical and geometrical conditions at the site of the emission left to informed guesswork. The linear polarisation of the emission provides a powerful additional diagnostic tool to constrain models of emission. However the measurement of $\gamma$-ray polarisation is no easy task. The detector systems are complex and unavoidably three dimensional in form and plagued by systematic errors, which are extremely difficult to evaluate. In turn this ensures that the analysis of the resulting data is an extremely difficult, complicated and time consuming process. Calibration is likewise very challenging.

Observations in the $\gamma$-ray regime allow us to scrutinize some of the most energetic emission processes associated with cosmic sources. Unlike most other wave bands that mainly see emission due to thermal reprocessing within hot gases, the majority of $\gamma$-ray emission is decidedly non-thermal in nature and is generally produced directly by highly energetic electrons in a well-ordered configuration, which involves particle beams and highly structured fields. Furthermore most of the relevant $\gamma$-ray production mechanisms (Synchrotron, Curvature, Inverse Compton and Bremsstrahlung) create polarised $\gamma$-rays with identifiable characteristics. The electrons which produce the $\gamma$-rays are often at the upper limit of the capability of the accelerator and due to their huge energy losses are unable to travel far from the source of injection as $\gamma$-ray emitters. Coupled with the extreme penetrating power of $\gamma$-ray photons this has the consequence that, through observing $\gamma$-rays, we are looking directly at the heart of the engine responsible for their acceleration. To produce electrons with Lorentz factors of $10^{7}-10^{8}$ or more requires an extremely ordered machine of well defined geometry. Such an arrangement is, quite naturally, ideal for the production of polarised radiation. Consequently the study of the polarisation characteristics of $\gamma$-ray emission provides an ideal means for the detailed understanding of the physics, geometry and inner working of the most powerful cosmic particle accelerators

Great strides have been made recently in the study of cosmic $\gamma$-ray sources through missions such as INTEGRAL, Swift and Fermi-LAT, and the discipline has moved from a few rudimentary measurements to the detailed studies of large numbers of cosmic sources. Although not designed as polarimeters the two main INTEGRAL instruments, SPI and IBIS are capable of studying the polarization characteristics of the stronger sources, and the first measurements are beginning to emerge for GRB 041219a [1], and the Crab nebula [2], [3].

\section{The Measurement of $\gamma$-ray Polarisation}

Measuring polarisation at $\gamma$-ray energies in the $100 \mathrm{keV}-10 \mathrm{MeV}$ range is usually accomplished using Compton scattering. The probability that the photon will scatter is given by the Klein-Nishina differential cross-section. In the case of a linearly polarised photon, this is given by 


$$
\frac{\boldsymbol{d} \boldsymbol{\sigma}}{\boldsymbol{d} \Omega}=\frac{\boldsymbol{r}_{0}^{2}}{2}\left(\frac{\boldsymbol{E}^{\prime}}{\boldsymbol{E}}\right)^{2}\left[\frac{\boldsymbol{E}^{\prime}}{\boldsymbol{E}}+\frac{\boldsymbol{E}}{\boldsymbol{E}^{\prime}}-2 \sin ^{2} \Theta \cos ^{2} \boldsymbol{\phi}\right]
$$

Where $r_{0}$ is the classical electron radius, $E^{\prime} / E$ is the ratio between the scattered photon and the incident photon energies and $\phi$ is the azimuthal scattering angle, defined as the angle to the polarisation electric vector. $\Theta$ is the elevation angle. [4] The secondary photon will preferentially scatter 90 degrees to the direction of electric vector. Measurement of the amplitude and azimuthal distribution of the scattered photons thus enables the degree of polarisation and vector direction of the polarised beam to be evaluated. The scattering takes place between detector pixels, and both the SPI and IBIS instruments on INTEGRAL can be used in this way. For the case of SPI scattering between adjacent Germanium detectors defines suitable events, whereas for IBIS the scatters take place between the ISGRI and PICSIT detection planes.

\section{INTEGRAL Crab Measurements}

INTEGRAL/SPI measurements of the Crab [2] have shown the emission to be highly polarised in the $\gamma$-ray domain, a measurement which has been independently confirmed using the entirely separate instrument INTEGRAL-IBIS [3]. Both sets of measurements were made at photon energies close to the electron rest mass, the most suitable for polarisation studies using Compton scatters. The SPI measurements were made in the "off-pulse" period (phase 0.5-0.8) and over the energy range $0.1-1.0 \mathrm{MeV}$, whereas the IBIS measurements for all the pulsar's phases and for energies above $0.2 \mathrm{MeV}$. The two independent polarisation measurements are in good agreement. The electric vector of off-pulse polarisation is found by SPI to be $123^{\circ} \pm 11^{\circ}$ (measured from north, anticlockwise on the sky), and by IBIS to be $122.0^{\circ} \pm 7.7^{\circ}$. There is no noticeable change in polarisation angle with phase. The percentage of polarisation has been found to be very high, in the case of SPI, this is reported to be $46 \pm 10 \%$, and the IBIS team report a value greater than $72 \%$ at the $95 \%$ confidence level. The IBIS team found no significant polarisation in the pulsed emission. As in the X-rays [5] both of the $\gamma$-ray observations encompass the entire nebula and pulsar, due to the angular resolution of the instruments involved.

In practice there are a number of systematic effects in the background noise that generate non-symmetrical azimuthal distributions and which can greatly compromise the polarisation sensitivity; e.g. for the case of the data set used for the SPI measurement [2], on a statistical basis the polarisation of the Crab was measurable to a precision of $0.03 \%$ [6], in practice systematic errors limited the accuracy to $\pm 10 \%$. Had the errors been solely statistical it would have been possible to measure the Crab polarisation with SPI to the 10\% level in around one minute! The $10 \%$ value was derived by combining the instrument data and results obtained from detailed Monte-Carlo Mass-Model (MM) simulations, [7] so as to use the MM to suppress the systematic contributions for all sources. Without the use of a MM the measurements would have been impossible. Even so there is clearly a long way to go; any errors in the MM likewise contribute to the systematic errors. 


\section{The Source of the Crab Emission}

The production of polarized emission provides an important diagnostic of the underlying physical system, but from where precisely do they originate? With a $\gamma$-ray emission spectrum of slope $\Gamma \sim 2.23$, the underlying electron spectrum will have a power law index $\mathrm{p} \sim 3.5$, which in turn constrains the maximum possible level of linear polarisation allowed by physics to be $\sim 77 \%$. [4]. With a measured $46 \%$ polarisation, the radiation clearly originates in an extremely well ordered machine. The best fit values for the angle of polarization for the non-pulsed soft $\gamma$ ray emission show a remarkable degree of alignment with the $124^{\circ} \pm 0.1^{\circ}$ direction of the spin axis of the neutron star projected onto the sky [10], and this is unlikely to be accidental. It is reasonable to assume that the spin axis of the neutron star in some way determines the geometry of the parent electron fluxes. Such a high degree of linear polarization is not compatible with any significant emission from the tangled magnetic fields associated with the Crab plerion. An inspection of radio polarization measurements [9] of this outer region demonstrates that a high degree of order does not exist there. The alignment with the spin axis and the high degree of polarization also implies that any significant production of highly energetic electrons does not take place within the nebula and must be generated close to the pulsar. It is also difficult to envisage how such a high net degree of polarization could result from the torus. As a consequence the bare polarization measurements demand that the soft $\gamma$-rays are generated somewhere inside the torus region or within the jet structure.

What about the energies of the parent electrons? Two key processes could be responsible for the emission: (i) Curvature radiation. Chandra images of the Crab [10] indicate that the jet is curved so energetic electrons streaming along the jet will radiate by curvature radiation with the electric vector parallel to the jet, as seen by INTEGRAL. In the relativistic limit, the emission frequency is given by $v_{c}=(3 c / 4 \pi R) \gamma^{3}$ where $\gamma$ is the Lorentz factor of the radiating electrons and $R$ is the radius of curvature of the field lines. Taking an estimate of $R \sim 2.5 \times 10^{18} \mathrm{~cm}$ for the $\mathrm{Crab}$ jet, in order to generate the few hundred $\mathrm{keV}$ photons detected by INTEGRAL, electrons with energies of typically $10^{15} \mathrm{eV}$ are required, which is entirely plausible in the context of the Crab pulsar. For radiation from within the inner jet, we would expect the direction of the electric vector associated with the polarised radiation to lie approximately parallel to the spin axis of the neutron star, as observed by INTEGRAL. (ii) Synchrotron radiation. The energies of the parent electrons are dependent upon the strength of the magnetic field in which they radiate, typical Crab B values in the range 100 to $1000 \mu \mathrm{G}$ lead to electron energies of $2-5 \times 10^{14} \mathrm{eV}$. For the case of a synchrotron mechanism the magnetic field must be orientated orthogonal to the spin axis of the pulsar. For both mechanisms, due to the high electron energies required and their associated short travel distances, the origin of the radiation is likely to be close to pulsar.

\section{Comparison with Optical Polarisation Measurements}

Polarization measurements of the Crab optical radiation have been reported by Slowikowska et al., [11] and previous references therein. The key measurement parameters for the non-pulsed optical emission are: a mean polarization angle of $\sim 123^{\circ}$ and a $33 \%$ degree of polarization. The angular resolution possible in the optical band enables the OPTIMA team to 
pin point the origin of the polarised optical emission to within about an arc second or so of the pulsar. The optical and $\gamma$-ray polarisation characteristics are remarkably consistent strongly suggesting that, for the non-pulsed radiation at least, both originate from the same region close to the pulsar and from a region well within the wind termination shock. The predicted polarization characteristics for mechanisms within the light cylinder, including the polar cap model, the outer gap model and the two pole caustic model have been extensively discussed in the literature [12], [13]. Whilst the different models have varying degrees of success in describing individual features, such as pulse profiles and pulse separations, none of these models can well reproduce the measured optical and $\gamma$-ray polarisation characteristics with the generally accepted values for the magnetic dipole offset angle $\left(\alpha \sim 60^{\circ}\right)$ and view angle $(\xi \sim$ $\left.61^{\circ}\right)[8]$.

The inclusion of the off pulse soft $\gamma$-ray polarization characteristics leads us to conclude that the soft $\gamma$-rays must be generated somewhere outside the pulsar's light cylinder and within the X-ray ring that marks the termination shock. Options include curvature radiation along inner jet, assumed aligned with spin axis, or synchrotron radiation in a field orthogonal to the spin axis. The bright optical knot discovered along the jet axis, 0.65 " southeast of the pulsar by Hester [14] lies within the OPTIMA error circle is a possible but unlikely source of the emission.

\section{The Striped Wind Model}

The very good correlation between the pulsar and PWN X-ray luminosities found by Kargaltsev and Pavlov [15] is not to be expected, unless the pulsed radiation comes not from the magnetosphere, but from the wind. [16] The idea of a striped pulsar wind was originally introduced by Coroniti [17], and has been extensively discussed in the literature. [18] This model places the site for the production of the pulsed and unpulsed radiation outside the light cylinder and within the termination shock. The energy transport is dominated by a Poynting flux with the pulsar driving a low frequency wave in the wind, consisting of stripes of toroidal magnetic field of alternating polarity propagating around the equatorial plane. The wind expands until the ram pressure is balanced by the surrounding nebula at the termination shock and the wave is dissipated. Relativistic beaming is responsible for the phase coherence of the synchrotron radiation, and is also responsible for the X-ray dark zone "observed" between the pulsar and the termination shock [10], the striped wind radiation thus appears to originate for an object of diminished angular size with a corresponding higher surface brightness. The striped wind model predicts that the magnetic field configuration for synchrotron radiation automatically aligns the polarization vector with the spin axis of the pulsar. This property is inherent to the striped wind model. The polarization characteristics expected from the striped wind model have been discussed in [19] and [18]. Generally the striped wind model provides a good description of both the optical and $\gamma$-ray observations for the Crab polarisation when the generally recognized Crab values of the magnetic dipole offset angle to the rotation axis of the pulsar and the angle of inclination of this rotating neutron star to the line of sight are included in the analysis. 


\section{The Vela Pulsar}

The near future scientific goals associated with the polarisation studies of pulsars in the context of currently available instrumentation are quite straightforward: it is to accumulate enough good quality on source data in order to identify the underlying mechanism for the production of the $\gamma$-rays and in particular the $\gamma$-ray polarization characteristics need to be measured and compared to available models. Of the 10 PWN systems detected by INTEGRAL, the Vela and MSH 15-52 systems provide the best opportunities for such tests. The first step will be to see if the $\gamma$-ray polarisation vector habitually aligns with the axis of rotation of the neutron star, such that the Crab value is not purely coincidental. Above we have seen that there are three contending scenarios, all of which are considered capable of explaining the alignment of the $\gamma$-ray polarisation vector with the pulsar spin axis, the subsequent challenge will be to identify which model best explains the detailed measurements.

Gamma-ray polarisation measurements above $\sim 50 \mathrm{MeV}$ were made of Vela by Caraveo et al. [21] using data from the Cos-B instrument. Their analysis was consistent with an extremely high $(\sim 100 \%)$ degree of linear polarisation having the electric vector aligned at a position angle of $130^{\circ} \pm 15^{\circ}$. It is of great interest to note that this direction is well aligned with the Vela jet position angle of $130^{\circ}$ measured with Chandra by Helfand, Gotthelf \& Halpern. [22].

\section{Future $\gamma$-Ray Polarisation Studies}

As outlined in the introduction most $\gamma$-ray sources are likely to exhibit strong polarisation characteristics, and furthermore polarisation measurements in this high energy band are likely to yield profound insights into the physics of the central energy source and its associated geometry.

The measurement of $\gamma$-ray polarisation from astrophysical jets in both the AGN and microquasar context is clearly of great interest. Bright extragalactic emitters such as Mkn421, 3C273, 3C279, will provide interesting and possibly fruitful targets for $\gamma$-ray polarisation studies. Radio observations demonstrate that highly structured magnetic fields exist and change throughout jet structures. Knots in the AGN structures also exhibit polarised radio emission, although the alignment bears little correlation with the jet structure. In both hard and soft states microquasar systems have power-law tails out to at least $1 \mathrm{MeV}$ and a maximum power output in the optimal 100's of keV $\gamma$-ray polarisation studies range [23], which must be associated with non-thermal particle populations and, by implication, the jets in these systems. See [24] for a review. A measure of the linear $\gamma$-ray polarisation degree and angle would provide information on the ordering and (re)orientation of the magnetic field in the jet very close to the black hole. However jet structures are not the only potential source of polarised high energy emission from accreting black hole systems. Theoretical studies have shown that we can expect to observe polarised X/ $\gamma$-ray emission from accretion disks [25] as well as from the corona associated with black holes [26]. Unified models for jet-disk coupling in black hole binaries (e.g. 24) explain the various states in terms of changing contributions from the jet, corona and accretion disk. Monitoring the $\gamma$-ray polarisation variations throughout the emission cycles would provide powerful insights to the transformations of underlying system. Oscillations between states in 
which the jet is ON/OFF should reveal differences attributable to a jet, whilst polarisation measurements in each state could reveal how the B-field near the black hole re-orders as the jet reforms. Among the best candidates are Cygnus X-1 and GRS 1915+105, both powerful jet sources where the jet orientation on the sky is well measured allowing for a direct comparison with any $\gamma$-ray polarisation angle.

However there are two key astrophysical characteristics that make the measurement of $\gamma$ ray polarisation challenging. (i) In both the extragalactic and galactic contexts $\gamma$-ray sources are notoriously variable on non-periodic timescales, dictating that measurements be made over a limited time period (but as outlined in the above paragraph it is of great astrophysical interest to achieve this aim). (ii) The degree of linear polarisation in jets, knots, accretion disks and coronae is expected to be only a few percent. From a measurement point of view these rather low levels are equivalent to greatly reduced source strengths. The net result for future $\gamma$-ray polarisation studies is that the polarised emissions will be considerably weaker and, due to the source variability, there will be a limited time period in which to effect the measurements. Higher polarisation sensitivity is therefore a must to advance from the current observational status in the field.

\section{8. $\gamma$-Ray Polarisation Studies - The Next Steps}

We have seen that it is probable that the majority of $\gamma$-ray emitting sources are likely to be polarised, and that polarised $\gamma$-ray photons carry with them vital information regarding the physical processes taking place in the most energetic machines within the universe together with a picture of the geometry within which the photons are generated. However a 10\% polarised source, for the purposes of measurement, is equivalent to a source of one tenth the emission strength; in turn this requires an observation period that is roughly one hundred times longer. The difficulties imposed by weak and time variable polarised emissions dictate that major improvements in the measurement instrumentation have to be made for progress to be achieved.

The current Crab measurements entailed the analysis of data accumulated over more than $10^{6}$ seconds, and the Crab is at least ten times stronger than the best polarised fluxes we can expect for the next crop of observations. However the Crab measurements have been dominated by systematic errors to the tune that the (systematic) error quoted for the Crab measurement was 300 times the error that would have been achieved on a statistical basis with a systematic free instrument. With a perfect instrument the published Crab value could have been attained in $10^{-5}$ of the time! The suppression of systematic errors is thus vital. Future instrumentation must be custom designed for polarimetry and not, as in the case of INTEGRAL happens to work as a polarimeter. Precise Mass Modelling is also crucial, but, further, future polarimeters should be evolved symbiotically with the Mass Modelling process to minimize systematics.

Improved instrumental sensitivity is also necessary, and the best way to achieve this is through some form of concentration of the incoming beam so as to enhance the signal to noise ratio. Note here that the $\gamma$-ray band around $1 \mathrm{MeV}$ is the only astronomical window where this does not happen. In this context the Laué lens technique currently offers the best possibility and recent studies [27] have demonstrated that the diffraction process does not distort the polarisation characteristics of the incoming beam for practical telescope arrangements. 


\section{References}

[1] S.McGlynn et al, 2007, Astronomy \& Astrophysics, 466, 895.

[2] A.J.Dean et al., 2008, Science, 321, 2008.

[3] M.Forot et al., 2008, The Astrophysical Journal, 688, L29.

[4] F.Lei, A.J.Dean \& G.L.Hills., 1997, Space Science Reviews, 82, 285.

[5] M.C.Weisskopf et al., 1978, The Astrophysical Journal, 220, 479.

[6] M.C.Weiskopf et al., 2006, The prospects for X-ray polarimetry and its potential use for understanding neutron stars, astro-ph/061148.

[7] A.J.Dean et al, 2003, Space Science Reviews, 105, 309.

[8] C.-Y.Ng \& R.W.Romani, 2004, The Astrophysical Journal, 601, L117.

[9] T.Velusamy, 1985, Monthly Notices of the Royal Astronomical Society, 212, 359.

[10] M.C.Weisskopf et al., 2000, The Astrophysical Journal, 536, L81.

[11] A.Slowikowska et al., 2009, Monthly Notices of the Royal Astronomical Society, 397, 103.

[12] J.Dyks, A.K.Harding, \& B.Rudak, 2004, The Astrophysical Journal, 607, 939.

[13] J.Takata et al., 2007, The Astrophysical Journal, 656, 1044.

[14] J.J.Hester et al., 1995, The Astrophysical Journal, 448, 240.

[15] O.Kargaltsev \& G.G.Pavlov, 2008, Pulsar Wind Nebulae in The Chandra Era, arXiv:0801.2602v2

[16] J.G.Kirk et al., 2002, Astronomy \& Astrophysics, 388, L29.

[17] F.V.Coroniti, 1990, The Astrophysical Journal, 349, 538.

[18] J.G.Kirk, et al., 2007, The Theory of Pulsar Wind Nebulae, astro-ph/0703116v1.

[19] J.Petri \& J.G.Kirk, 2005, The Astrophysical Journal, 627, L37.

[20] J.Petri\& Y.Lyubarsky, 2007, Astronomy \& Astrophysics, 473, 683.

[21] P.A.Caraveo, et al. 1985, The Astrophysical Journal, 327, 203.

[22] D.J.Helfand, E.V.Gotthelf \& J.P.Halpern, 2001, The Astrophysical Journal, 556, 380.

[23] M.L.McConnel et al., 2000, The Astrophysical Journal, 543, 928.

[24] R.P.Fender, T.M.Belloni \& E.Gallo, 2004, Monthly Notices of the Royal Astronomical Society, $\mathbf{3 5 5}$, 1105 .

[25] R.A. Sunyaev \& L.G. Titarchuk, 1985, Astronomy \& Astrophysics, 143, 374.

[26] J.D. Schnittman \& J.H Krolik, 2010, The Astrophysical Journal, 712, 908.

[27] R.M.Curado da Silva et al. 2009, Polarimetry Study with a CdZnTe Focal Plane Detector, Preprint. 